\title{
Mouse Genome Informatics
}

National Cancer Institute

\section{Source}

National Cancer Institute. Mouse Genome Informatics. NCI Thesaurus. Code C48299.

A resource of the Jackson lab, provides integ rated access to data on the genetics, genomics, and biology of the laboratory mouse. 